\title{
Embarazos forzados: ser madre antes de los quince
}

\author{
(1) Mónica Gogna \\ Conicet/Universidad de Buenos Aires, Facultad de Filosofía y Letras, Instituto Interdisciplinar- \\ io de Estudios de Género
}

\section{Georgina Binstock \\ Conicet/Centro de Estudios de Población}

\section{Introducción ${ }^{1}$}

Desde los primeros estudios realizados en la década de 1980, el embarazo y la maternidad en la adolescencia continúan siendo temas de interés de la política pública debido a la persistencia de niveles relativamente altos de fecundidad y a que la mayoría de estos embarazos son reportados como no intencionales, lo que supone una vulneración a los derechos sexuales y reproductivos de las adolescentes (Binstock y Gogna, 2014). Como en otros países de la región, la baja "deseabilidad" de los embarazos en esta etapa de la vida está relacionada con una cultura que no reconoce la condición de sujetos sexuales de las/os adolescentes y no facilita su acceso a anticonceptivos eficaces ni al aborto seguro (Rodríguez Vignoli, 2014).

Particularmente crítica resulta, por un conjunto de razones, la maternidad en las menores de quince años. Por una parte, muchos de estos embarazos son producto de abusos sexuales (Chiarotti, 2016; Planned Parenthood-Global, s./f.), con las devastadoras consecuencias que ello supone para la salud psíquica y emocional de una niña (Jeejebhoy y Bott, 2003). A su vez, las niñas de catorce años o menos tienen el mayor riesgo de complicaciones y muerte por el embarazo y el parto (UNFPA, 2013). ${ }^{2}$ No menos importantes son las potenciales consecuencias de la maternidad temprana sobre la vida cotidiana de la adolescente y sus posibilidades de desarrollo futuro.

1 El presente artículo resume parte de los hallazgos del "Estudio sobre maternidad adolescente con énfasis en menores de 15 años”, impulsado y financiado por UNICEF Argentina y coordinado por Georgina Binstock y Mónica Gogna desde el Centro de Estudios de Población (CENEP). Agradecemos a Cecilia Velázquez y Daniela Rey (asistentes), a Liliana Ensisa y Lidia Schiavoni (coordinadoras de campo), a las entrevistadoras y muy especialmente a las adolescentes que compartieron con nosotras sus experiencias. Nuestro reconocimiento a Fernando Zigman (especialista en Salud, UNICEF) y a Julián Govea Basch (consultor) por sus comentarios al informe final de investigación. Los resultados y opiniones expresadas en este artículo son de exclusiva responsabilidad de las autoras.

2 En el caso de la Argentina, Pantelides, Marconi y Fernández (2014), advierten que si bien la escasa cantidad de casos llama a ser prudentes en las afirmaciones, la mortalidad materna es más alta entre las adolescentes menores. 
Asimismo, en los hijos de menores de quince años es entre quienes se encuentran los mayores porcentajes de nacimientos pretérmino y con bajo o muy bajo peso al nacer. Las tasas de mortalidad fetal y mortalidad infantil son significativamente más altas entre las menores de quince con respecto a las madres de las demás edades (Pantelides, Marconi y Fernández, 2014; ASUMEN, s./f.)

La cantidad de partos de adolescentes menores de quince años de edad en la Argentina varió entre 2.500 y 3.400 casos anuales desde el año 2000 a la fecha. En 2017, se registraron 2.787 nacimientos: casi el $60 \%$ de ellos acontecieron en 5 de las 24 jurisdicciones de nuestro país: Buenos Aires (23,7\%); Santa Fe (10,3\%); Chaco, Salta y Misiones (entre 7,4 y $8,2 \%$ ) (DEIS, 2018).

A su vez, en 2013 (último año informado), se registraron 533 egresos hospitalarios de niñas de entre 10 y 14 años de edad internadas por complicaciones de abortos, lo que constituye un dato preocupante y una cuestión de la que sabemos poco, más allá de su magnitud (DEIS, 2015).

No obstante su relevancia en términos de salud pública y derechos humanos, la problemática de la maternidad precoz está invisibilizada, con excepción de esporádicas noticias periodísticas - generalmente de tono sensacionalista - acerca de las "madres niñas".

Un punto de inflexión en el tratamiento de la temática en la prensa escrita fue el editorial del diario La Nación "Niñas madres con mayúsculas" (01/02/19). A pocos días de que en Jujuy se realizara una cesárea a una niña que había solicitado la interrupción legal de un embarazo producto de violación, el editorial celebraba la decisión de dos niñas de 13 y 14 años de continuar sus embarazos (uno de ellos producto de violación y el otro de una relación que no quedaba claro si había sido consentida) y tildaba de "criminales" a sus madres, por aconsejarles la interrupción, a la que las niñas tenían derecho en el contexto legal vigente.

El texto, que sostenía la existencia del "instinto materno", recibió un repudio unánime e instantáneo en las redes sociales y en los medios de comunicación. Legisladores de diferentes partidos políticos, periodistas (incluidos los de La Nación), organismos gubernamentales y no gubernamentales, Unicef Argentina, Amnistía Internacional y la Campaña por el Derecho al Aborto Legal, Seguro y Gratuito se pronunciaron con firmeza en contra de los conceptos allí vertidos. Esa misma noche, el diario publicó los comunicados de Unicef y Fundación Huésped. "El embarazo en la infancia no está vinculado al 'instinto materno', es abuso sexual y por tanto el embarazo es forzado. Los adultos (familia, Estado, instituciones) son responsables de proteger a las niñas y niños frente al abuso sexual" (Unicef Argentina). "La maternidad forzada es una forma de tortura y suma una violación más a los derechos de estas niñas" (Fundación Huésped).

El diario también aclaró que las notas editoriales representaban exclusivamente la posición editorial de la empresa y que lamentaba que el texto se hubiera interpretado "como un mensaje de alguna forma tolerante hacia los abusos a menores, algo que, como el mismo editorial señalaba, es a todas luces repudiable".

\section{Objetivos, marco conceptual y metodología}

Nuestro estudio tuvo por objetivo producir conocimiento acerca de una problemática que, por diversos motivos (su baja incidencia, la carencia de datos oficiales, la 
sensibilidad de la temática), solo recientemente ha comenzado a ser objeto de investigación social en la región y en nuestro país. La Argentina fue uno de los catorce países latinoamericanos incluidos en el estudio pionero coordinado por Chiarotti en 2016. Con anterioridad Pantelides, Marconi y Fernández (2014) habían realizado un análisis sociodemográfico y sanitario de las madres menores de quince años en base a datos de las estadísticas vitales y el sistema informático perinatal.

Este artículo reporta resultados de un estudio cuanti-cualitativo realizado en 2016 en tres provincias (Misiones, Chaco y Buenos Aires) impulsado y auspiciado por Unicef Argentina.

En términos conceptuales, adoptamos la definición de embarazo infantil forzado propuesta por Chiarotti: "se considera embarazo infantil forzado cuando una niña (menor de 14) queda embarazada sin haberlo buscado o deseado y se le niega, dificulta, demora u obstaculiza la interrupción del embarazo". El embarazo puede ser producto de una violación sexual o provenir de una relación sexual consensuada, en la que la niña no conocía las consecuencias o, conociéndolas, no pudo prevenirlas. Es el caso de niñas que no han tenido educación sexual, o habiéndola tenido no pudieron acceder a métodos de prevención de los embarazos y de anticoncepción de emergencia (Chiarotti, op.cit.).

A partir de la información de la DEIS (Dirección de Estadísticas e Información de Salud), del análisis del registro de nacimientos de 2016 (último disponible a la fecha de la elaboración de este artículo) ${ }^{3}$ y del examen de los datos del Censo de población y viviendas-2010, realizamos un diagnóstico sociodemográfico que provee el contexto necesario para una mejor comprensión de los resultados del estudio cualitativo. Asimismo, actualiza estudios similares (Giusti y Pantelides, 1991; Binstock y Pantelides, 2005) por lo que es posible establecer cambios y continuidades vinculados a las conductas reproductivas adolescentes, así como indicar brechas en las desigualdades que imprime la maternidad temprana en el curso y condiciones de vida de las adolescentes.

También realizamos diecinueve entrevistas semiestructuradas con adolescentes que habían sido madres antes de los quince años y una entrevista con una adolescente de catorce años embarazada. Las adolescentes fueron contactadas vía instituciones de salud (Hospital del Bicentenario en Castelli, Chaco), referentes barriales (GBA y Posadas), la Defensoría de Niños, Niñas y Adolescentes (Misiones) y Hogares (Hogar de Día y Hogar San Francisco en Posadas). La muestra incluyó dos adolescentes de la etnia qom y una de la etnia wichi. Del total de contactos realizados, se registraron cuatro rechazos.

Las entrevistas tenían por objetivo conocer las historias de las adolescentes, el contexto de sus embarazos, sus reacciones y las de sus entornos, si habían considerado la posibilidad de interrumpirlos, sus experiencias de acceso a servicios de salud y de protección y justicia (en los casos en que correspondía), las consecuencias de los embarazos y las maternidades sobre la escolaridad y sobre sus vidas cotidianas y sus expectativas a futuro. Como es de rigor, se solicitó a las adolescentes su consentimiento informado. Las entrevistas, realizadas por una de las investigadoras principales y por entrevistadoras con experiencia, fueron grabadas, previa autorización de las adolescentes.

3 Se refiere a la base de datos y a la posibilidad de realizar cruces de datos. Cuando estuvieran disponibles se incluyen datos de 2015 que fueran recientemente publicados. 


\section{La fecundidad temprana: tendencias y brechas}

En la Argentina la tasa de fecundidad tardía ( 15 a 19 años) descendió de manera lenta pero continua desde 80 nacimientos por mil adolescentes de esas edades en 1980 hasta 56,7 por mil en 2003, fecha en que comenzó a ascender, alcanzando en 2014 un valor de 65,1 por mil (Ministerio de Salud, 2016). En 2015 se observó un leve descenso (62,6 por mil), que se habría intensificado en 2016 (55,9 por mil) y en 2017 (53,1 por mil) según los datos preliminares disponibles (DEIS, 2015. 2016. 2017). Es necesario esperar a ver si esta tendencia a la baja se confirma.

A fines comparativos, cabe señalar que la Argentina se ubica actualmente por encima del promedio mundial, estimado en 48,9 nacimientos por cada mil mujeres de 15 a 19 años, pero por debajo de la media de América Latina y el Caribe, de 73,2 por mil.

La distribución de la maternidad adolescente tiene una estrecha relación con el nivel de desarrollo: en términos generales, a mayor nivel de desarrollo corresponde menor fecundidad. Si bien las brechas entre jurisdicciones se han reducido, aún persisten importantes diferencias que van desde el 26,3 por mil en la Ciudad Autónoma de Buenos Aires hasta el 101,2 en Chaco; 95,2 en Misiones; 92,8 por mil en Formosa; y 83,9 por mil en Salta (DEIS, 2015).

Respecto de la repetición del embarazo durante la adolescencia, esta disminuyó durante el primer quinquenio de la década de 2000 (del 31\% al 26\% entre las adolescentes de 18-19 años) y se ha mantenido estable en dicho valor. Chaco, Misiones, Santiago del Estero y Entre Ríos son las provincias con la proporción más alta de segundos nacimientos o más: alrededor de uno de cada tres nacimientos de adolescentes mayores (18 y 19 años).

Con respecto a la fecundidad temprana (10-14 años), esta es actualmente de 1,5 por mil y presenta también variaciones marcadas entre jurisdicciones que van del 0,4 por mil en la Ciudad de Buenos Aires al 5,2 en Chaco. Misiones, Formosa, Salta y Corrientes tienen tasas de fecundidad precoz mucho más elevadas que el promedio (entre 3,7 y 4,7 por mil).

En 2017, en la Argentina se registraron 94.709 nacimientos de madres menores de veinte años, los que representan el $13,5 \%$ del total de nacimientos. Los nacimientos de madres menores de quince años fueron 2.493 en 2017 y representaron el 2,6\% del total de los nacimientos adolescentes (y el o,35\% del total de nacimientos). La mayoría de los nacimientos de madres precoces corresponde a las de catorce años y una proporción no desdeñable a quienes tienen trece o doce años. En 2014, último año para el que se dispone de información desagregada, dicha proporción alcanzaba el $18 \%$ y, si tenemos en cuenta el volumen, representa 470 niñas que dan a luz a los trece años y 65 a los doce años, lo que refleja una situación de extrema vulnerabilidad. En términos de su perfil sociodemográfico, el $44,5 \%$ de las madres menores de quince años convivía con su pareja al registro del nacimiento y más de la mitad (55\%) de ellas tenía primaria completa o menos educación.

Uno de los rasgos a los que siempre se asocia la maternidad adolescente, particularmente la precoz, es a los riesgos de salud, entre ellos una mayor prevalencia de nacimientos de bajo peso y de prematurez (variables que a su vez están asociadas entre sí).

Los datos más recientes disponibles muestran que aún persisten diferencias y son las madres precoces quienes tienen el mayor porcentaje de hijos con bajo peso (10,3\%), proporción que desciende conforme aumenta la edad, siendo 7,5\% entre las adolescentes de dieciocho y diecinueve años, y $7 \%$ entre las madres adultas. Si se compara 
esta información con la de una década atrás, se observa un descenso de la proporción de nacimientos de bajo peso, particularmente entre las adolescentes y entre aquellas de menor edad. También entre las menores de quince años disminuyó la proporción de nacidos de menos de $1,500 \mathrm{~kg}$ (de 2,6 a 2,3\% en 2005 y 2014, respectivamente). Estos resultados en conjunto parecen apuntar a una mejora en la atención de las adolescentes menores, ya sea a partir de un diagnóstico de embarazo más temprano y/o mejor seguimiento en su atención médica que repercuten en un incremento de sus niños con el peso adecuado.

En cada edad, la proporción de madres entre quienes viven en hogares con NBI duplica a la de sus pares que viven en hogares sin NBI. A los catorce años, es del casi $3 \%$ entre las que tienen NBI versus $1,5 \%$ entre las que no. Las brechas son también evidentes cuando se aborda la pobreza en función de los ingresos del hogar. El $2 \%$ del quintil 5 ha tenido un hijo antes de los quince años mientras que en el quintil 1 no se registró ninguna adolescente en dicha situación (y solo el o,2\% en el quintil 3) (Plan-Unicef, 2014). La maternidad antes de los quince también es más baja entre las argentinas (1,6\% es madre) que entre sus pares extranjeras (entre el 4,0 y 4,7\%).

Datos del último censo indican que entre las adolescentes de catorce años, casi la totalidad de quienes no son madres (95\%) asiste a un establecimiento educativo mientras que entre las madres la proporción desciende al 66\% (lo que representa una brecha del $45 \%$ ). La condición de actividad es otro de los rasgos en que se diferencian las adolescentes madres de las no madres, particularmente a edades precoces. Entre las adolescentes de catorce años, el 30\% de las adolescentes madres son económicamente activas mientras que entre quienes no son madres desciende a la mitad (14\%).

\section{La maternidad temprana en primera persona. Hallazgos del compo- nente cualitativo}

Entre los factores asociados a la maternidad precoz se encuentran las características del hogar de la adolescente: el ingreso de sus progenitores, sus niveles de educación y la condición de pobreza del hogar (Plan-Unicef, 2014). La gran mayoría de las entrevistadas pertenece a familias de nivel socioeconómico bajo o muy bajo (viviendas precarias, bajo nivel educativo de los padres, trabajos eventuales o de muy baja calificación). Sus madres tuvieron su primer/a hijo/a en la adolescencia y la mayoría de ellas ha tenido una numerosa cantidad de hijos. Solo cuatro entrevistadas vivían con sus parejas al momento del encuentro (siendo tres de ellos los padres biológicos de sus hijos). En general, las adolescentes vivían con sus hijos y con uno o ambos progenitores y varios hermanos. Eran minoría quienes vivían con otros familiares (abuela, abuelos, tía) o en hogares para madres adolescentes. En este último caso encontramos indicios similares a los del estudio regional: aunque sus necesidades básicas estén cubiertas, las sensaciones de aislamiento y prisión pueden ser difíciles de sobrellevar. Son situaciones sobre las que debería profundizarse en estudios futuros, en especial por tratarse de casos judicializados en los que intervienen los organismos de protección de derechos.

Un cuarto de las niñas entrevistadas estaba fuera de la escuela al momento del embarazo. Los motivos de abandono fueron la necesidad de trabajar o hacerse cargo de hermanos. En otros dos casos se debió a una convivencia temprana o a la "prohibición del novio" (veintitrés años mayor). La mayoría de las que estaban asistiendo, por su parte, dejaron la escuela por motivos similares a los reportados en el estudio regional. "Como que tenía vergüenza después de ir con la panza... De eso sí tenía vergüenza." 
(Melina, Misiones). "Hablaban porque era chiquita y porque... no sé, hablaban nomás, así de mí... (...) me choca mucho lo que dicen y ahí dejé." (Paula, Misiones)

Otras mencionaron circunstancias relacionadas con el embarazo en sí (tenía que caminar mucho para llegar a la escuela, le costaba subir las escaleras; etc.). Algunas entrevistadas reportaron actitudes de acompañamiento y cuidado por parte de directivos y docentes y otras, episodios de discrecionalidad respecto de la normativa destinada a facilitar la continuidad de los estudios a las alumnas embarazadas o madres.

Luego del parto, el amamantamiento y el cuidado del bebé dificultan la vuelta a la escuela. Al momento de las entrevistas, tres adolescentes habían retomado la escuela. La única que completó el secundario pudo hacerlo cuando su hijo tenía ya un año. Al igual que se observó en el estudio regional, quienes retomaron sus estudios lo hicieron mediante módulos domiciliarios o escuelas nocturnas, en las que las adolescentes parecen sentirse más cómodas.

Algo más de la mitad de las entrevistadas había tenido al menos una charla de educación sexual, mayoritariamente después de su iniciación sexual. La mayoría indicó que había hablado con la madre acerca de la menstruación y de "cuidarse", generalmente a instancias de la madre ya que ellas no se animaban a preguntar o les daba vergüenza. Los profesionales de salud no jugaron un rol importante en la información y entrega de métodos anticonceptivos sino hasta después del embarazo.

Las niñas que reportaron haberse iniciado sexualmente de forma consensuada lo hicieron mayoritariamente con un "novio". ${ }^{4}$ Con respecto a la edad, la mayoría se inició a los trece ( 2 a los doce, 2 a los once y una a los diez). Ocho de los varones eran adolescentes (13 a 19 años) y el resto las superaba ampliamente en edad (21, 24, 40 y 50 años). Para algunas la iniciación sexual fue un evento inesperado. No fueron infrecuentes los testimonios del estilo: "muchos nervios", "muy así...de sorpresa", "tenía miedo. No había pensado en eso".

Otras, en cambio, se iniciaron luego de algunos meses de relación y, una adolescente indicó que le había pedido al novio que le diera tiempo hasta que "se animara". No parece haber sido la situación de Brenda (GBA), quien manifiesta que hubiera preferido esperar. La mitad de las entrevistadas reportaron haberse cuidado en la iniciación sexual, conducta que se abandonó rápidamente o se volvió poco sistemática. ${ }^{5}$

En la mayoría de los casos de esta muestra intencional, los embarazos fueron producto de relaciones que se reportaron como consentidas. ${ }^{6}$ En varios de ellos, sin embargo, la importante diferencia de edad entre la niña/adolescente y su pareja sexual permite inferir la existencia de una desigualdad de poder en la toma de decisiones respecto de las relaciones sexuales, el cuidado anticonceptivo y el deseo de maternidad/paternidad. Julieta (GBA) tuvo un hijo a los trece con un varón de veintiún años (el novio de su tía). Cecilia, madre a los catorce, se embarazó producto de una relación que tuvo a los trece con un vecino de veintiún años. Ana convivió desde los doce años con un hombre de aproximadamente cuarenta, con quien tuvo dos hijos - el primero a los trece años (actualmente están separados y ambos tienen nuevas parejas). Quizás el 4 No se obtuvo información sobre iniciación sexual en el caso de las niñas cuyos embarazos fueron producto de violaciones. En uno de ellos, se infiere que fue la primera relación sexual y en el otro (una niña perteneciente a la etnia wichi) no se preguntó directamente pues la madre estuvo en la entrevista y fue quien contestó la mayoría de las preguntas.

5 Solo una entrevistada afirma haberse cuidado "siempre" y atribuye su embarazo a que el preservativo debió estar "pinchado".

6 En el estudio de Planned Parenthood (s./f.), el 80\% de los embarazos de la muestra de Perú se reporta como producto de relaciones consentidas. Un estudio sobre madres de 10 a 14 años en Chile indica que "la mayoría de las adolescentes menores de 14 años tienen relaciones sexuales voluntarias y en gran proporción con otro menor de edad” (Molina et al., 2007). 
caso más impactante sea el de Lara (madre a los trece, Misiones) quien tiene una hija de seis meses fruto de una relación con un hombre de alrededor de cincuenta años. Según relató, ella quería tener un hijo porque su mamá había muerto. En la entrevista manifestó: "Le dije ‘sí, yo quiero un hijo, por ahí me ayuda más"”.?

Los embarazos se produjeron en el contexto de uniones tempranas o relaciones cortas, definidas como noviazgos por las entrevistadas, en las que no se usaron métodos anticonceptivos, se los usó mal o, a juicio de las entrevistadas, los métodos fallaron. Al traducir los dichos de una adolescente qom, la intérprete describe una situación que puede alcanzar a otras adolescentes menores de quince años, más allá de su pertenencia étnica. "(...) si, eh, la primer relación que ellos tuvieron, si, se cuidaron pero después dejó de cuidarse, como que ella era muy chica y no entendía tampoco... que nunca pensó que iba a quedar así... embarazada". (Lucía, Chaco, nuestro énfasis).

En otros casos, fueron los varones quienes decidieron que no se usaría un método anticonceptivo. Carla (Misiones) reporta que se cuidaban "a veces" ya que su novio le decía "no pasa nada". Gabriela sostiene "después dejamos, porque a él no le gustaba, no se sentía cómodo con eso (con preservativo) y yo tampoco". Andrea (Misiones), embarazada al momento de la entrevista, dejó las pastillas porque su pareja no quería que las tomara.

También el tabú en torno a la sexualidad adolescente puede constituir un obstáculo para el uso de un método anticonceptivo. Es el caso de Eugenia (GBA) quien conocía las pastillas anticonceptivas pero que no fue a la salita a buscarlas porque la madre, a quienes todos conocen en el barrio, no sabía que ella "estaba con él". Se reportaron también dificultades atribuidas a métodos en mal estado ("pastillas vencidas", "preservativos pinchados").

Como señala el estudio regional, también se registraron embarazos producto de violación. En un caso, el violador (padrastro) cumplía su condena y la niña y su hija vivían con una tía. En otro (violación de una niña wichi por parte de un criollo) al momento de la entrevista se aguardaba el resultado del ADN. En otros dos casos, existía la sospecha de embarazo producto de abuso sexual (una adolescente que vivía en el campo y no había revelado la identidad del padre de su bebé y otra que vivía con sus abuelos y asistía a un hogar de día).

Todas las situaciones descriptas están contenidas en la definición de embarazo infantil forzado, el que proviene de una relación sexual forzada o de una relación consensuada en que la niña no conocía las consecuencias de la actividad sexual o, conociéndolas, no pudo prevenirlas. La falta de acceso a una educación sexual temprana e integral, las relaciones desiguales de poder entre los géneros, la falta de servicios de salud amigables $^{8} \mathrm{y}$ la pobreza las expusieron a una maternidad temprana no buscada.

Con excepción de las dos entrevistadas que vivían en hogares para madres, que indicaron haberse sentido contentas, al resto la noticia le provocó fundamentalmente miedo y desconcierto. Las reacciones de los padres de las adolescentes abarcaron un amplio espectro (enojo, "no tan bien al principio", "contentos") pero no fueron masivamente negativas como podría esperarse dadas sus cortas edades. En dos casos, los padres sugirieron el aborto, que luego no se concretó. Una vez confirmado el

7 El otro caso de embarazo reportado como buscado fue el de Paula, quien tuvo relaciones con su novio, dos años mayor que ella, y no se cuidó. “... esa vez no me cuidé a propósito, porque yo estaba reenamorada de él y lo quería solo para mí, y... no sé qué me pasó”.

8 Se entiende por servicios de salud amigables aquellos que entregan un conjunto de servicios esenciales para los adolescentes, tienen proveedores motivados y capacitados que saben cómo comunicarse con los usuarios, garantizan la confidencialidad y trabajan en el marco de consentimiento informado. (UNFPA, 2005). 
embarazo, algunas entrevistadas comenzaron rápidamente a recibir mensajes que las instaban a asumir el rol materno: [que tenía que] "hacerse cargo del bebé", "tenés que ocuparte de la criatura".

Con algunas pocas excepciones, la mayoría de las parejas sexuales se desentendieron del embarazo (o estuvieron con ellas durante unos pocos meses más) y no mantenían vínculos con sus hijos/as al momento de las entrevistas.

Aun cuando desde el punto de vista legal, todas hubieran calificado para una interrupción legal del embarazo, ya fuera por la causal violación o por la causal salud -el embarazo y el parto antes de los quince años son causa de mayor morbimortalidad perinatal y materna (ASUMEN, s./f.)-, obstáculos de diverso tipo dificultaron el acceso al aborto no punible. Entre ellos, el desconocimiento de la normativa vigente, el descubrimiento tardío del embarazo, el estigma asociado al aborto y la presión social y/o de personas del entorno cercano.

Sí, se me cruzó la idea, antes de contarle a mi mamá. Una amiga me había dicho que yo tome un remedio para no tenerlo. Ella me había dado. Un yuyo. Y cuando estaba hirviendo mi mamá me preguntó por qué estaba por tomar eso, si estaba embarazada. Ahí me encerré en la pieza. Estaba por abortar. Yo no sabía qué hacer. Tenía miedo. (Cecilia, Misiones)

Y ahí yo ya hablo con mi pareja y yo no quería, no quería, no quería [continuar el embarazo] y mi mamá no sabía nada y yo le decía que yo no quería y él me dijo que "no podemos, no vamos a echarlo porque sería feo algo asi". Y ahí yo pensé y después muchas personas me dijeron que era feo echarlo, que ya es tuyo y que es una persona, dijeron. (Melina, Misiones)

La noción de que el embrión es una persona también estuvo presente en el discurso de otras dos entrevistadas que en algún momento contemplaron la posibilidad del aborto pero luego la desestimaron.

Solo una entrevistada reconoció haber intentado abortar con medicamentos, pero sin conseguirlo. "No quería saber nada. Estaba por abortar pero no pude... yo tomé pastillas, me puse pastillas, todo. Pero no lo despedía al bebé. Y bueno, después sentí que no lo quería hacer. Mi mamá me quería comprar más pastillas para... pero bueno. Era muy chica, no quería tener todavía un bebé” (Eugenia, GBA).

\section{Conclusiones y recomendaciones}

Recapitulando, los datos cuantitativos muestran que son las niñas más pobres quienes se convierten en madres antes de los quince años. Las historias ilustran un "entretejido" de factores detrás de esas realidades: la pobreza, la aceptación de las uniones tempranas por parte de las familias y las comunidades, la desescolarización, la violencia sexual, la falta de una educación sexual temprana (que permita establecer relaciones de género más igualitarias, prevenir el abuso sexual y comunicarlo a adultos confiables cuando ocurra), la ausencia de servicios de salud accesibles y amigables.

Aun cuando en solo dos de los veinte casos analizados el embarazo fue con certeza producto de violación, en los restantes aplica - en sentido amplio- la noción de embarazo forzado, pues las niñas/adolescentes no tenían conciencia de las consecuencias de la actividad sexual o no tuvieron las herramientas necesarias para prevenir un embarazo que mayoritariamente no buscaron y que las angustió y asustó. Aquellas 
que hubieran querido interrumpir esa gestación tampoco pudieron acceder a esta práctica. Como señala el informe de CLADEM, frente a esta realidad es imperioso que los Estados implementen planes, políticas y medidas sociales para la prevención de los embarazos y las maternidades infantiles forzados y la adecuada sanción de la violencia sexual que los provoca (Chiarotti, 2016).

A continuación, algunas recomendaciones - surgidas de los hallazgos del estudio y de la literatura regional - que pueden ser de utilidad para pensar acciones que resguarden los derechos de las niñas y adolescentes. Evitar la deserción escolar de las niñas en situación de vulnerabilidad es un eje clave para prevenir y evitar tanto situaciones de abuso como embarazos no deseados (Santillán, Neufeld y Cerletti, 2007). Cuando estos ya han ocurrido, la escuela tiene el deber de acompañar y orientar a las niñas ante situaciones de violencia, identificar tempranamente las situaciones de embarazo, ofrecer información y facilitar el acceso a la interrupción legal del embarazo. Para ello, es fundamental la existencia de una articulación previa entre la institución escolar y el centro de salud. En tal sentido, resulta necesario documentar las experiencias de las incipientes asesorías escolares que han comenzado a funcionar en algunas provincias y evaluarlas a los fines de que puedan ser replicadas.

La mayoría de las entrevistadas abandonó la escuela producto del embarazo. Ello muestra que la normativa vigente destinada a garantizar la continuidad escolar de las adolescentes embarazadas y madres es insuficiente. Deberían implementarse mecanismos que faciliten la continuidad escolar. Existen experiencias tanto en la ciudad como en la provincia de Buenos Aires que podrían servir de inspiración. Se trata de iniciativas que contemplan la figura de un/a referente escolar. En su rol de articuladores con otras instituciones (centros de salud, organismos de protección de derechos), los/as referentes pueden facilitar las intervenciones apropiadas en los casos en que se detecten situaciones de abuso o embarazos no buscados.

Si tenemos en cuenta que las niñas están asistiendo a la escuela al momento de iniciarse sexualmente, la implementación de la ESI desde el nivel inicial resulta una tarea impostergable (Binstock y Naslund-Hadley, 2010). Implementar la ESI en todo el país con docentes capacitados y motivados resulta vital para que las/os adolescentes conozcan sus derechos, cuenten con más recursos para denunciar los abusos y establezcan tempranamente relaciones de género más igualitarias.

Resulta necesaria la articulación entre la escuela y el centro de salud, para facilitar y estimular el acceso a la provisión de atención, consejería y anticoncepción (incluyendo la anticoncepción hormonal de emergencia). Una situación de gran vulnerabilidad que requiere una atención especial, es la de las niñas y adolescentes indígenas que no tienen la posibilidad de ser comprendidas en su propio idioma. Resulta prioritario establecer alguna estrategia (por ejemplo, la formación e incorporación de agentes sanitarios bilingües) con el objetivo de lograr un mayor acercamiento entre las comunidades y los centros de salud que permita "superar la barrera lingüística y acercar universos culturales muy distintos" (Menna, 2010).

Entre las recomendaciones para evitar y reducir los embarazos precoces, la OMS propone permitir a las adolescentes obtener servicios de aborto seguro (Chiarotti, 2016). Implementar la estrategia de reducción de riesgos y daños 9 y facilitar el acceso a la interrupción legal del embarazo a las niñas y adolescentes menores debería ser parte de una estrategia integral destinada a proteger sus derechos y promover sus oportunidades de vida.

9 Esta estrategia "consiste en que el profesional desaliente a la paciente a que incurra en prácticas peligrosas para su salud y que la aconseje para que, si tomó la decisión de terminar su embarazo, lo haga de la manera más segura posible" (Zamberlin, 2013). 
Asimismo resulta prioritario trabajar en la prevención del abuso sexual. Esto supone tanto la realización de campañas e intervenciones destinadas a visibilizar la problemática del abuso y desnaturalizar la violencia como que el Estado actúe con eficacia y celeridad frente a denuncias de abuso sexual y brinde protección integral a las víctimas.

Prevenir el embarazo precoz supone evitar la asunción temprana de roles adultos por parte de las/os adolescentes y aumentar sus posibilidades de completar los estudios y acceder así a mejores oportunidades de vida. Alcanzar este objetivo depende en gran medida de con qué esfuerzos programáticos e inversión de recursos la sociedad se organice para ofrecerles condiciones que les permitan efectivamente ejercer sus derechos (Ayres et al., 1998) y alcanzar sus sueños. 


\section{Q Referencias bibliográficas}

» ASUMEN-Alianza Argentina para la Salud de la Madre, Recién Nacido y Niño. (s./f.). Llegar tarde en los embarazos tempranos: una emergencia social

» Ayres, J. R. et. al. (1998). Vulnerabilidade do Adolescente ao HIV/AIDS. En Vieira, E. M.; Fernandes, M. E. L.; Bailey, P. y MacKay, A (orgs.). Seminário Gravidez na adolescência. Río de Janeiro, Associação Saúde da Família.

»Binstock, G. y Gogna, M. (2014). Entornos del primer y segundo embarazo en la adolescencia en Argentina. En Cavenaghi, S.y Cabella, W. (eds.). Comportamiento reproductivo y fecundidad en América Latina: una agenda inconclusa. Serie e-Investigaciones. núm. 3, pp. 167-185. Río de Janeiro, FNUAP-ALAP.

»------ (2017). Estudio sobre maternidad adolescente con énfasis en menores de 15 años. Informe final. CENEP.

» Binstock, G. y Naslund-Hadley, E. (2010). Iniciación sexual, asistencia escolar y embarazo adolescente en sectores populares de Asunción y Lima: una aproximación cualitativa. En Debates en Sociología, núm. 35. Lima, Fondo de la Editorial Pontificia Universidad Católica del Perú.

»Binstock, G. y Pantelides, E. A. 2005. La fecundidad adolescente hoy: diagnóstico sociodemográfico. In: M. Gogna (Coord.), Embarazo y maternidad en la adolescencia. Estereotipos, evidencias y propuestas para políticas públicas, CEDES/ UNICEF/ Ministerio de Salud de la Nación, Buenos Aires: 77-112.

»Chiarotti, S. (coord.). (2016). Niñas madres. Embarazo y maternidad infantil forzada en América Latina y el Caribe. Balance regional. CLADEM.

»Dirección de Estadísticas e Información de Salud-DIES. (2013). Indicadores seleccionados de salud para población de 10 a 19 años. República Argentina-Año 2011. En Boletín 141-DEIS. Buenos Aires, Ministerio de Salud, Presidencia de la Nación Argentina.

»----- (2016). Estadísticas Vitales Información Básica. Año 2015. Serie 5, núm. 59. DEIS. Buenos Aires, Ministerio de Salud, Presidencia de la Nación Argentina.

》------- (2017). Estadísticas Vitales Información Básica. Año 2016. Serie 5, núm. 6o. DEIS. Buenos Aires, Ministerio de Salud, Presidencia de la Nación Argentina.

»------ (2018). Vitales Información Básica. Año 2017. Serie 5, núm. 61. DEIS, Ministerio de Salud y Desarrollo Social, Presidencia de la Nación Argentina.

» Giusti, A. y Pantelides, E. A. (1991). Fecundidad en la adolescencia. República Argentina 1980-1985. Buenos Aires, Ministerio de Salud y Acción Social, Dirección de Estadísticas de Salud, Serie 8, núm. 11.

» Jejeebhoy, S. y Bott, S. (2003). Non Consensual sexual experiences in young people: A review of the evidence from developing countries. Population Council.

" Menna, F. (2010). La mujer indígena en la Argentina: un nuevo sujeto que emerge en la agenda pública. Trabajo presentado en el Congreso Internacional Las políticas de equidad de género en perspectiva: nuevos escenarios, actores y articulaciones. Área Género, Sociedad y Política. FLACSO.

»Ministerio de Salud de la Nación (2016). Situación de salud de los y las adolescentes en la Argentina. Buenos Aires. 
" MOLINA C, Ramiro; MOLINA G, Temístocles y GONZALEZ A, Electra. Madres niñas-adolescentes de 14 años y menos: Un grave problema de salud pública no resuelto en Chile. Rev. méd. Chile [online]. 2007, vol.135, n.1 [citado 2020-06-17], pp.79-86. Disponible en: <https://scielo.conicyt.cl/scielo. php? script=sci_arttext\&pid=So034-98872007000100011\&lng=es\&nrm=iso $>$. ISSN 0034-9887. http://dx.doi.org/10.4067/So034-98872007000100011.

»Pantelides, E. A.; Fernández, M. y Marconi, E. (2014). Maternidad temprana en la Argentina. Las madres menores de 15 años. Trabajo aceptado para su presentación en las XIII Jornadas Argentinas de Estudios de Población, Salta, 16 al 18 de septiembre. Buenos Aires, CENEP-Ministerio de Salud.

»Plan International-UNICEF (2014). Salinas Mudler, S. (coord.) Vivencias y relatos sobre el embarazo en adolescentes. PANAMA.

"Planned Parenthood. (s./f.). Vidas robadas: un estudio multipaís sobre los efectos en la salud de las maternidades forzadas en niñas de 9-14 años.

"Rodríguez Vignoli, J. (2014). La reproducción en la adolescencia y sus desigualdades en América Latina. Introducción al análisis demográfico con énfasis en el uso de microdatos censales de la ronda de 2010. Santiago de Chile, CEPAL-UNFP.

»Santillán, L.; Neufeld, M. R.y Cerletti, L. (2007). Las familias en la Escuela. En Serie Pedagogía, Programa de capacitación multimedial. Buenos Aires, Ministerio de Educación, Ciencia y Tecnología, Presidencia de la Nación.

» UNFPA-Equipo de Apoyo Técnico de América Latina y el Caribe. (2005). Capacitación en derechos y salud sexual y reproductiva de adolescentes en América Latina y el Caribe. México DF.

"Williamson, N. et al. (2013). Maternidad en la niñez. Enfrentar el reto del embarazo en adolescentes. UNFPA.

"Zamberlin, N. 2013. Reducción de riesgos y daños asociados al aborto inseguro. II Conferencia Subregional CLACAI Cono Sur. El aborto en el Cono Sur: Avances hacia el acceso al aborto seguro y legal. Buenos Aires, 4 y 5 de noviembre. FUSA-CLACAI. 